\title{
Correction to: The effect of beta blocker withdrawal on adenosine myocardial perfusion imaging
}

\author{
C. Hoffmeister, ${ }^{a}$ R. Preuss, MD, ${ }^{b}$ R. Weise, ${ }^{b}$ W. Burchert, MD, PhD, ${ }^{b}$ and \\ O. Lindner, $M D, P^{b}{ }^{b}$
}

a Diabetes Center, Heart and Diabetes Center North Rhine-Westphalia, University Hospital of the Ruhr-University Bochum, Bad Oeynhausen, Germany

b Institute of Radiology, Nuclear Medicine and Molecular Imaging, Heart and Diabetes Center North Rhine-Westphalia, University Hospital of the Ruhr-University Bochum, Bad Oeynhausen, Germany

doi: $10.1007 / \mathrm{s} 12350-019-01593-3$

\section{CORRECTION TO: JOURNAL OF NUCLEAR CARDIOLOGY ${ }^{\circledR}$ VOLUME 21, NUMBER 6;1223-9 HTTPS://DOI.ORG/10.1007/S12350-014-9952-Y}

Regrettably the original version of the above article contained errors in Table 2 and wrong values in the text. The corrected table is presented here and the values which have been corrected now appear in bold text.

Page 1223 Abstract

Global MBF showed an increase from $180.2 \pm 59.9$ to $193.6 \pm 60.8 \mathrm{~mL}$ minute $/ 100 \mathrm{~g}(P=\mathbf{. 0 0 2})$ after beta blocker withdrawal.

\section{Page 1225}

Mean systolic and mean diastolic blood pressure during adenosine were nearly identical $(P=.77$ and $P=.79)$ with and without beta blocker. Mean heart rate and mean RPP during adenosine significantly increased after beta blocker withdrawal by $\mathbf{1 5 . 2} \% \pm 17 \%$ $(P=001)$ and $\mathbf{1 6 . 2} \% \pm 23 \%(P=.004)$, respectively.

Page 1226

The data are listed in Table 2, lower third. Global MBF showed a significant increase by $7.4 \% \pm 10 \%$ $(P=.002)$ after beta blocker withdrawal. The individual

The original article can be found online at https://doi.org/10.1007/ s12350-014-9952-y.

Reprint requests: C. Hoffmeister, Diabetes Center, Heart and Diabetes Center North Rhine-Westphalia, University Hospital of the Ruhr-

University Bochum, Bad Oeynhausen, Germany; clhoffmei@gmx.de J Nucl Cardiol 2019;26:688-9.

1071-3581/\$34.00

Copyright (C) 2019 American Society of Nuclear Cardiology. 
Table 2. Hemodynamic response under adenosine, perfusion, and left-ventricular function

\begin{tabular}{|c|c|c|c|c|c|}
\hline & $\begin{array}{l}\text { With beta } \\
\text { blocker }\end{array}$ & $\begin{array}{l}\text { Without beta } \\
\text { blocker }\end{array}$ & $\Delta$ & $(\Delta \%)$ & $\boldsymbol{P}$ \\
\hline Heart rate (BPM) & $69.7 \pm 12.1$ & $80.3 \pm 10.9$ & 10.6 & 15.2 & $<.001$ \\
\hline Systolic blood pressure (mmHg) & $117.3 \pm 19.9$ & $118.2 \pm 19.3$ & .9 & .8 & .77 \\
\hline Diastolic blood pressure $(\mathrm{mmHg})$ & $55.8 \pm 9.9$ & $56.1 \pm 8.8$ & .3 & .5 & .79 \\
\hline Rate-pressure product & $8159.5 \pm 1943.0$ & $9487.0 \pm 2025.4$ & 1327.5 & 16.2 & .004 \\
\hline $\mathrm{EDV}(\mathrm{mL})$ & $164.5 \pm 36.5$ & $16.6 \pm 43.9$ & 1.9 & 1.1 & .59 \\
\hline ESV $(m L)$ & $61.8 \pm 12.6$ & $62.9 \pm 16.0$ & 1.1 & 1.7 & .64 \\
\hline $\mathrm{EF}(\%)$ & $39.4 \pm 10.7$ & $40.6 \pm 0.3$ & 1.2 & 3.0 & .29 \\
\hline $\begin{array}{l}\text { Global myocardial perfusion (mL/minute/ } \\
100 \mathrm{~g})\end{array}$ & $180.2 \pm 59.9$ & $193.6 \pm 60.8$ & 13.4 & 7.4 & .002 \\
\hline $\begin{array}{l}\text { Minimal coronary resistance }(\mathrm{mmHg} /(\mathrm{mL} / \\
\text { minute/100 g)) }\end{array}$ & $0.49 \pm 0.2$ & $0.45 \pm 0.2$ & -.04 & -8.1 & .038 \\
\hline $\begin{array}{l}\text { Global perfusion related to RPP (mL/ } \\
\text { minute/100 g) }\end{array}$ & $229.6 \pm 96.7$ & $206.0 \pm 73.1$ & -23.6 & -10.2 & .032 \\
\hline
\end{tabular}

data are depicted in Figure 1. All but three patients had a lower global MBF without beta blocker than with. The segmental MBF values (Figure 2) demonstrated a strong correlation over the entire range of perfusion values. The average effect was a slight perfusion shift of about $1015 \mathrm{~mL}$ minute- $1 / 100 \mathrm{~g}$ in the range of 100 $300 \mathrm{~mL}$ minute- $1 / 100 \mathrm{~g}$. The $\mathrm{mCR}$ under adenosine declined by $8.1 \% \pm 11 \%(P=.038)$ and the normalized RPP by $16.2 \% \pm 21 \%(P=\mathbf{. 0 0 4})$ after betablocker discontinuation

Disclosure Oliver Lindner was supported by Carinopharm. 